\title{
Association of Cerebrospinal Fluid Parameters and Neurofilament Light Chain With Retinal Nerve Fiber Layer Thickness in Multiple Sclerosis
}

OPEN ACCESS

Edited by:

Jodie Burton,

University of Calgary, Canada

Reviewed by:

Shiv Saidha,

Johns Hopkins Medicine,

United States

Grigorios Kalaitzidis,

Johns Hopkins Medicine,

United States

*Correspondence:

Gabriel Bsteh

gabriel.bsteh@meduniwien.ac.at

†These authors have contributed equally to this work and share first authorship

Specialty section:

This article was submitted to

Multiple Sclerosis and

Neuroimmunology,

a section of the journa

Frontiers in Neurology

Received: 14 November 2021

Accepted: 31 January 2022

Published: 07 March 2022

Citation:

Krajnc N, Altmann P, Riedl K, Mitsch C, Berger T, Leutmezer F,

Rommer P, Pemp B and Bsteh G (2022) Association of Cerebrospinal Fluid Parameters and Neurofilament Light Chain With Retinal Nerve Fiber Layer Thickness in Multiple Sclerosis. Front. Neurol. 13:814734.

doi: 10.3389/fneur.2022.814734

\author{
Nik Krajnc ${ }^{1+}$, Patrick Altmann ${ }^{1+}$, Katharina Riedl ${ }^{1}$, Christoph Mitsch ${ }^{2}$, Thomas Berger ${ }^{1}$, \\ Fritz Leutmezer ${ }^{1}$, Paulus Rommer ${ }^{1}$, Berthold Pemp ${ }^{2}$ and Gabriel Bsteh ${ }^{1 *}$ \\ ${ }^{1}$ Department of Neurology, Medical University of Vienna, Vienna, Austria, ${ }^{2}$ Department of Ophthalmology, Medical University \\ of Vienna, Vienna, Austria
}

Introduction: Multiple sclerosis (MS) pathophysiology comprises both inflammatory and neurodegenerative characteristics. Cerebrospinal fluid (CSF) analysis allows for assessment of inflammation while neurofilament light chain can indicate neuroaxonal damage. Retinal thinning is a robust prognostic biomarker for neurodegeneration in MS. To date, an association between CSF parameters upon MS diagnosis and retinal thinning has not been investigated.

Aims and Objectives: We aimed to determine whether CSF parameters are associated with the evolution of retinal layer thinning in people with MS (pwMS).

Methods: For this longitudinal observational study, we investigated pwMS from the Vienna MS database (VMSD), who had undergone (1) a diagnostic lumbar puncture (LP) between 2015 and 2020, and (2) simultaneous optical coherence tomography (OCT) and/or (3) a follow-up OCT scan. Linear stepwise regression models were calculated with OCT parameters (peripapillary retinal nerve fiber layer [pRNFL] thickness at LP and at follow-up, annualized loss of pRNFL thickness [aLpRNFL]) as a dependent variable, and CSF parameters (white blood cell [WBC] count, total protein [CSFTP], CSF/serum albumin ratio $\left[Q_{a l b}\right]$, intrathecal synthesis of immunoglobulins, neurofilament light chain [NfL] in both CSF and serum [CSFNfL/sNfL]) as independent variables adjusted for age, sex, and disease duration.

Results: We analyzed 61 pwMS (median age 30.0 years [interquartile range 25.5-35.0], $57.4 \%$ female, median disease duration 1.0 month [IQR 0-2.0] before LP, median follow-up 1.9 years [IQR 1.1-3.5]). CSFNfL and sNfL measurements were available in 26 and 31 pwMS, respectively. PRNFL thickness at LP was inversely associated with the CSF WBC count $(\beta=-0.36 ; 95 \% \mathrm{Cl}-0.51,-0.08 ; p=0.008)$. We did not find any association between other CSF parameters, including CSFNfL, sNfL, and aLpRNFL.

Conclusions: Increased WBC count as an indicator of acute inflammation and blood-brain-barrier breakdown seems to be associated with the amount of retinal thickness already lost at the time of LP. However, neither routine CSF parameters nor a singular NfL measurement allows the prediction of future retinal thinning.

Keywords: multiple sclerosis, biomarker, optical coherence tomography, RNFL, cerebrospinal fluid, neurofilament 


\section{INTRODUCTION}

Multiple sclerosis (MS) is an inflammatory demyelinating disease of the central nervous system (CNS) that represents the most common neurological disease in young adults (1). The current prevailing concept of MS pathophysiology suggests a disease process of both inflammatory and neurodegenerative characteristics. While neurodegenerative processes are predominating in later phases, it seems that neurodegeneration starts early on and, thus, influences long-term prognosis. Hence, there is an urgent need for surrogate markers that allow for the reliable evaluation of MS-associated neurodegeneration throughout the disease course as well as at the time of diagnosis (2). Current concepts for measuring neuroinflammation and neurodegeneration upon MS diagnosis include optical coherence tomography (OCT), standard cerebrospinal fluid (CSF) parameters, and neurofilament light chain (NfL). OCT is a non-invasive and accessible technique that uses near-infrared light to create high-resolution cross-sectional images of retinal layers (3). The thickness of the peripapillary retinal nerve fiber layer (pRNFL) and the macular ganglion cell-inner plexiform layer (GCIPL) are both robust indicators of neuroaxonal degeneration in MS (4). Annualized loss of both pRNFL (aLpRNFL) and GCIPL (aLGCIPL) thickness exceeding 1.5 and $1.0 \mu \mathrm{m} /$ year, respectively, predict disability progression at considerable sensitivity and specificity (5-7). CSF analysis is a standard diagnostic procedure in the initial evaluation of MS in Europe. The leukocyte count (white blood cell [WBC] count, cell count) in the CSF as a non-specific indicator of acute inflammation is increased up to 50 cells $/ \mu$ l in about half of people with MS (pwMS) (8). The hallmark of typical CSF changes in MS is the increased production of intrathecal immunoglobulins (Ig), indicated by an increased IgG index or the presence of CSF oligoclonal bands (OCB) (9-11). Over the past few years, neurofilament light chain (NfL) in the blood and CSF have emerged as a promising biomarker in MS (12). As a major component of the neuronal cytoskeleton, $\mathrm{NfL}$ is released into the CSF and blood upon neuroaxonal injury (13). NfL concentrations are associated with the occurrence of relapses and neurological disability as well as MS lesions and brain atrophy on MRI $(13,14)$.

To date, no studies have been published concerning the prognostic impact of the described CSF parameters on retinal layer thinning. With this study, we investigated whether

\footnotetext{
Abbreviations: aLGCIPL, annualized loss of ganglion cell-inner plexiform layer; aLpRNFL, annualized loss of peripapillary retinal nerve fiber layer; ARR, annualized relapse rate; CIS, clinically isolated syndrome; CSF, cerebrospinal fluid; $\mathrm{CV}$, coefficient of variance; DMT, disease modifying treatment; EDSS, Expanded Disability Status Scale; ESC-DMT, escalation of disease-modifying treatment; GCIPL, ganglion cell-inner plexiform layer; H-DMT, highly effective disease-modifying treatment; IQR, interquartile range; LP, lumbar puncture; MDMT, moderately effective disease-modifying treatment; MS, multiple sclerosis; NfL, neurofilament light chain; OCB, oligoclonal bands; OCT, optical coherence tomography; ON, optic neuritis; pRNFL, peripapillary retinal nerve fiber layer; pwMS, patients with multiple sclerosis; Qalb, CSF/serum albumin ratio; SD, standard deviation; TP, total protein; VMSD, Vienna Multiple Sclerosis Database; WBC, white blood cell.
}

conventional CSF parameters and NfL are associated with pRNFL at baseline and follow-up in a cohort of newly diagnosed pwMS.

\section{METHODS}

\section{Patients and Definitions}

For this longitudinal observational study, we extracted serum and CSF from patients in the Vienna MS database (VMSD) diagnosed with relapsing-remitting MS (RRMS) according to the 2017 McDonald criteria, aged between 18 and 65 years, who had a diagnostic lumbar puncture (LP) between January 1, 2015 and December 31, 2019, and (1) OCT at LP, and/or (2) follow-up OCT.

We documented age and disease duration at baseline as well as the duration of the follow-up period, defined by an interval between the diagnostic LP and the last OCT scan. A relapse was defined as patient-reported symptoms and objectively confirmed neurological signs typical of an acute CNS inflammatory demyelinating event with a duration of at least $24 \mathrm{~h}$ in the absence of fever or infection and separated from the last relapse by at least 30 days (15). The Expanded Disability Status Scale (EDSS) score was obtained at baseline and follow-up (16), with EDSS progression defined as a confirmed EDSS increase of $\geq 1.5$ points in patients with a baseline EDSS of 0 , an increase of $\geq 1.0$ point in patients with a baseline EDSS of 1.0-5.5, or an increase of $\geq 0.5$ point in patients with a baseline EDSS of $\geq 6.0$.

The patients' disease-modifying treatment (DMT) status was classified as follows: (1) "no DMT" defined as patients receiving no DMT between diagnostic LP and the last OCT scan; (2) "moderately effective DMT (M-DMT)" defined as patients receiving either interferon-beta preparations, glatiramer acetate, dimethyl fumarate or teriflunomide; or (3) "highly effective DMT (H-DMT)" defined as patients receiving either natalizumab, fingolimod, alemtuzumab, cladribine, ocrelizumab, or rituximab. We also defined "escalation (ESC-DMT)" as patients switching from no DMT to moderately effective DMT or from moderately effective DMT to highly effective DMT between the diagnostic LP and the last OCT scan.

\section{CSF Parameters}

In all patients, routine LP was performed at baseline as part of the routine diagnostic work-up following written informed consent. The routine CSF parameters included: WBC count, total protein ( $\mathrm{CSF} T \mathrm{TP}), \mathrm{CSF} /$ serum albumin ratio $\left(Q_{\mathrm{alb}}\right)$, intrathecal synthesis of IgA, IgM, and IgG including oligoclonal-IgG-bands (OCB).

\section{Optical Coherence Tomography}

Optical coherence tomography (OCT) imaging was performed by experienced neuro-ophthalmologists at the Department of Ophthalmology and Optometry of the same institution using the same spectral-domain OCT (Spectralis OCT, Heidelberg Engineering, Heidelberg, Germany; software Heidelberg eye explorer software version 5.4.8.0) without pupil dilatation in a dark room on both eyes of each patient. For pRNFL measurement, a custom $3.4 \mathrm{~mm}$ ring scan $\left(12^{\circ}\right)$ centered on the optic nerve head was used (1,536 A-scans, automatic real-time tracking [ART]: 100 averaged frames) (17), Image processing was 
conducted semiautomated with manual correction of obvious errors. All examinations were checked for sufficient quality using the OSCAR-IB criteria (18). For patients without a history of optic neuritis (ON), pRNFL thickness was calculated as the mean of the values for both eyes. For patients with a history of unilateral $\mathrm{ON}$, only the values of eyes without $\mathrm{ON}$ were used in the analyses.

Eyes suffering $\mathrm{ON}$ during the observation period were excluded from the longitudinal part of the study and only the values of eyes without $\mathrm{ON}$ during the observation period were used for the calculation of retinal thinning in the analyses (6). To identify subclinical $\mathrm{ON}$ at baseline, we used interocular asymmetry with cut-off values of $\geq 5 \mu \mathrm{m}$ for pRNFL $(19,20)$. To identify subclinical ON, during the course of the study, we used interocular asymmetry in retinal thinning (i.e., an increase in the inter-eye difference in PRNFL compared to the prior OCT) with a cut-off value of $\geq 5 \mu \mathrm{m}$. In these cases, we used only the eye with the higher value. aLpRNFL was calculated by individual linear regression models as the slope of the regression line best fitter to all pRNFL measurements over the observation period $(6,21)$. Based on the previous studies, we dichotomized patients into two groups using a pRNFL cut-off value of $88 \mu \mathrm{m}$ and an aLpRNFL cut-off value of $1.5 \mu \mathrm{m} /$ year $(6,21)$. Patients with diagnoses of ophthalmological, neurological, or drug-related causes of vision loss or retinal damage not attributable to MS were excluded (22). The investigators performing the OCT were blinded to clinical parameters and vice versa. The quantitative OCT study results were reported using the revised Advised Protocol for OCT Study Terminology and Elements (APOSTEL 2.0) recommendations (23).

\section{Neurofilament Light Chains}

Serum and CSF samples were extracted from our neurological biobank where aliquots are stored at $-70^{\circ} \mathrm{C}$ in accordance with international consensus guidelines $(24,25)$. Concentrations of sNfL and CSFNFL were measured on the SR-X analyzer (Quanterix International, Billerica, MA, USA) using commercially available Simoa NF-light kits and following the manufacturer's instructions. The investigators performing the ${ }_{\mathrm{CSF}} \mathrm{NfL}$ and sNfL testing were blinded to the clinical and OCT parameters, and vice versa. The samples were analyzed as duplicates. Only samples yielding a coefficient of variance (CV) of $<0.2$ were included in this study. Sensitivity analyses for missing NfL values were performed as a separate analysis of parameters for subjects with available and subjects with missing NfL values.

\section{Ethics}

The study was approved by the ethics committee of the Medical University of Vienna, Austria (EK1446/2021).

\section{Statistics}

Statistical analysis was performed using SPSS 26.0 (SPSS Inc, Chicago, IL, USA). Categorical variables were expressed in frequencies and percentages, continuous variables as mean and $\mathrm{SD}$ or median and interquartile range (IQR) as appropriate. Continuous variables were tested for normal distribution by the Kolmogorov-Smirnov test with Lilliefors correction. Univariate comparisons were done by a chi-square test, Mann-Whitney $\mathrm{U}$-test, or independent $t$-test as appropriate.

First, we performed a univariate analysis with Pearson and Spearman correlation analyses on the CSF parameters (WBC, CSF TP, $Q_{\mathrm{alb}}$, intrathecal synthesis of IgA, IgM and IgG, OCB) and OCT parameters (pRNFL at LP and at last follow-up, aLpRNFL). Linear stepwise regression models were fitted with the OCT parameters (pRNFL, aLpRNFL) as the dependent variable and the CSF parameters as independent variables adjusted for age, sex, and disease duration. Second, the CSF NfL, sNfL, and OCT parameters were univariately analyzed by Pearson or Spearman correlation analyses. Linear stepwise regression models were fitted with the OCT parameters as the dependent variable, and the CSF parameters, CSF NfL, and sNfL as independent variables (in separate models to avoid collinearity) adjusted for age, sex, and disease duration. As covariates, the number of relapses and disability progression during follow-up as well as the DMT group was included. A value of $p<0.05$ was considered statistically significant. All multiple analyses were corrected using the Bonferroni method.

\section{RESULTS}

\section{Demographics}

We included 61 pwMS in the analysis (Figure 1). In total, 53 (86.9\%) had a baseline OCT scan, 38 (62.3\%) had a follow-up OCT scan, and $25(41.0 \%)$ had both a baseline and a followup OCT scan. Baseline CSF samples for ${ }_{\mathrm{CSF}} \mathrm{NfL}$ measurements were available in $26(42.6 \%)$ patients, and baseline serum samples for sNfL measurements were available in 31 (50.8\%) patients. Sensitivity analyses regarding missing NfL values did not show a significant effect of missing values on the reported results. The demographics and clinical characteristics of the study cohort are given in Table 1. Oligoclonal bands (OCB) were positive in 58 patients, 2 patients were negative for $\mathrm{OCB}$, and the $\mathrm{OCB}$ results for one patient were missing. At baseline, no patient received DMT.

\section{pRNFL at Baseline}

The mean pRNFL thickness at baseline was $97.0 \mu \mathrm{m}$ ([SD] $=10.9)$. In total, $11(20.8 \%)$ patients had a pRNFL thickness $\leq 88 \mu \mathrm{m}$ at baseline. We detected no differences in the CSF parameters between these two groups. In the multivariable analyses, pRNFL thickness at baseline was associated with the WBC count $(\beta=-0.36$; $95 \%$ CI $-0.51,-0.08 ; p=0.008)$ but not with any of the other CSF parameters (Figure 2).

\section{pRNFL at Follow-Up}

The mean pRNFL thickness at follow-up was $95.6 \mu \mathrm{m}$ (SD = 10.0). In total, 7 (18.4\%) patients had a pRNFL thickness $\leq 88 \mu \mathrm{m}$ at follow-up. We detected no differences in CSF parameters between these two groups, and there was no association between pRNFL thickness and CSF parameters.

\section{aLpRNFL}

The median follow-up period was 1.9 (IQR 1.1-3.5) years. The mean aLpRNFL was -0.6 (SD 1.5) $\mu \mathrm{m}$ /year with 6 (24.0\%) 


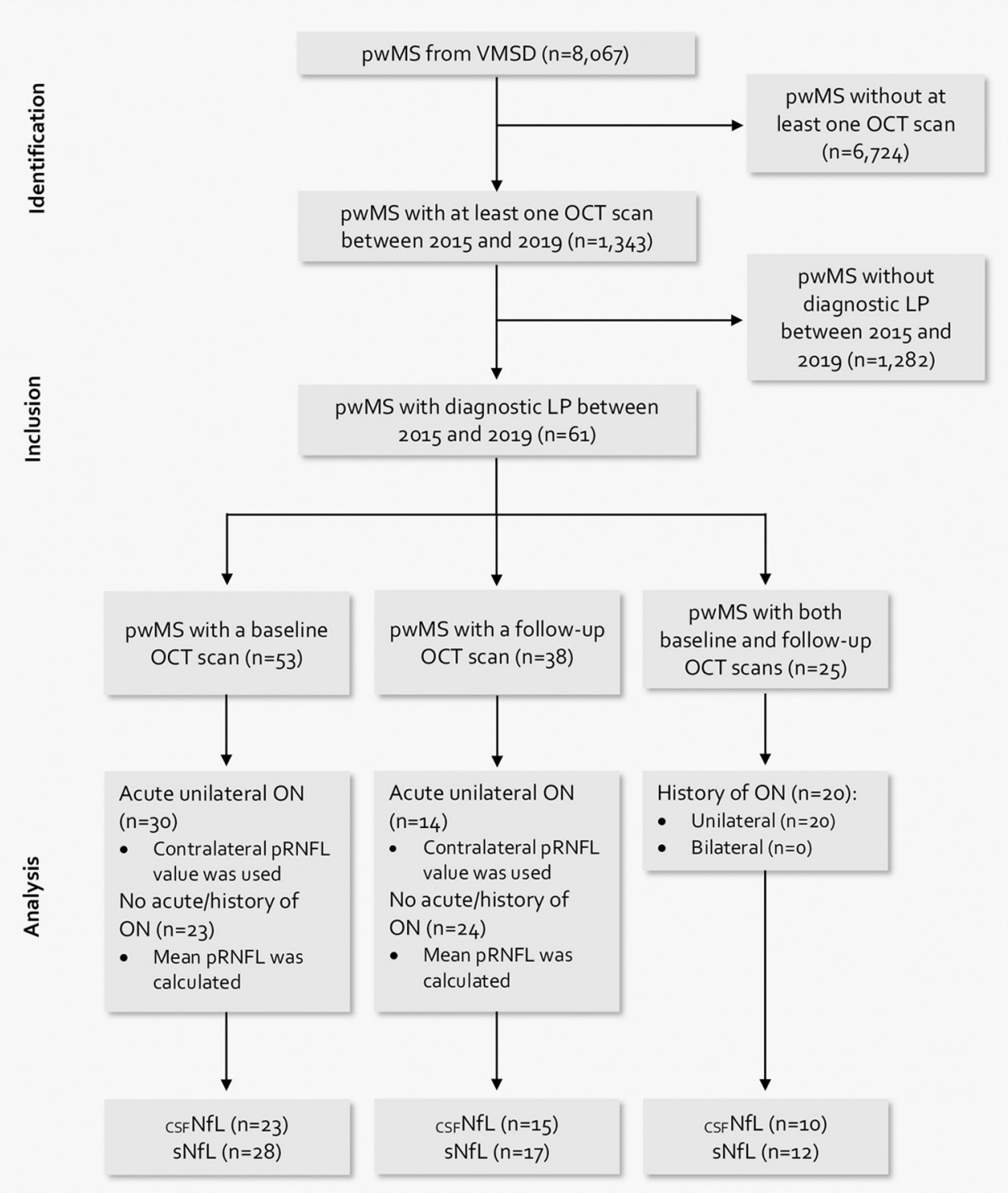

FIGURE 1 | Flow chart of patient selection based on the inclusion and exclusion criteria.

patients showing an aLpRNFL $\geq 1.5 \mu \mathrm{m}$ /year. In our sample, the aLpRNFL correlated with both the IgA ratio $\left(r_{s}=0.629, p\right.$ $=0.016)$ and IgA index $\left(r_{s}=0.678, p=0.008\right)$ but not with other CSF parameters (Table 2). Multivariate regression models revealed no association between CSF parameters and aLpRNFL.

\section{NfL Concentrations}

The median NfL concentrations at baseline were $798.8 \mathrm{pg} / \mathrm{ml}$ (IQR 426.3-1,507.6) for CSF and $10.6 \mathrm{pg} / \mathrm{ml}$ (IQR 7.1-20.2) for serum. As expected, CSF NfL correlated significantly with sNfL $\left(r_{s}=0.785 ; p<0.001\right)$; however, no correlation with $\mathrm{pRNFL}$ or aLpRNFL was found. Patients with evidence of neuroaxonal loss (characterized by an aLpRNFL $\geq 1.5 \mu \mathrm{m} /$ year) had higher concentrations of ${ }_{\mathrm{CSF}} \mathrm{NfL}$ (830.7 vs. $497.3 \mathrm{pg} / \mathrm{ml}$ ) and sNfL (6.9 vs. $8.0 \mathrm{pg} / \mathrm{ml}$ ) compared to patients with aLpRNFL $<1.5 \mu \mathrm{m} /$ year, but these differences were not statistically significant ( $p=0.999$ and $p=0.933$, respectively) (Figure 3 ). We also analyzed NfL concentrations for patients below and above the cohort's median pRNFL thickness of $88 \mu \mathrm{m}$ but found no significant difference between these groups (Figure 4).

\section{DISCUSSION}

In this longitudinal observational study of 61 pwMS, we aimed to determine an association between conventional and novel CSF parameters at the time of diagnosis and OCT parameters at baseline and follow-up (median 1.9 years). This is important, as retinal layer thinning on OCT is a relevant biomarker for MSassociated neurodegenerative changes, such as neuroaxonal loss, physical and cognitive disability, and brain atrophy $(22,26-28)$. 
TABLE 1 | Demographics and clinical characteristics of the study cohort.

\begin{tabular}{|c|c|c|c|c|}
\hline & Study cohort $(n=61)$ & Baseline cohort $(n=53)$ & Follow-up cohort ( $n=38$ ) & aLpRNFL cohort $(n=25)$ \\
\hline Female $^{a}$ & $35(57.4)$ & $29(54.7)$ & $24(63.2)$ & $13(52.0)$ \\
\hline Age (years) ${ }^{b}$ & $30.0(25.5-35.0)$ & $29.0(24.3-32.5)$ & $29.5(26.0-37.0)$ & $27.0(25.0-34.5)$ \\
\hline Follow-up period (years) $)^{b}$ & NA & NA & $1.9(0.9-3.3)$ & $1.9(1.1-3.5)$ \\
\hline EDSS at baseline ${ }^{b}$ & NA & $1.0(0-2.0)$ & NA & $0.5(0-1.9)$ \\
\hline $\mathrm{ARR}^{\mathrm{b}}$ & NA & NA & $0.8(0.6-1.2)$ & $0.8(0.5-1.4)$ \\
\hline EDSS progression during follow-upa & NA & NA & $9(23.7)$ & $7(28.0)$ \\
\hline \multicolumn{5}{|l|}{ DMT } \\
\hline No DMTa & $31(50.8)$ & $53(100.0)$ & $9(23.7)$ & $7(28.0)$ \\
\hline M-DMTa & $21(34.4)$ & $0(0)$ & $20(52.6)$ & $11(44.0)$ \\
\hline H-DMT'a & $9(14.8)$ & $0(0)$ & $8(21.1)$ & $7(28.0)$ \\
\hline Fingolimod & $2(3.3)$ & NA & NA & NA \\
\hline Natalizumab & $2(3.3)$ & NA & NA & NA \\
\hline Alemtuzumab & $1(1.6)$ & NA & NA & NA \\
\hline Rituximab & $3(4.9)$ & NA & NA & NA \\
\hline Ofatumumab & $1(1.6)$ & NA & NA & NA \\
\hline ESC-DMTa & $1(1.6)$ & $0(0)$ & $1(2.6)$ & $0(0.0)$ \\
\hline \multicolumn{5}{|l|}{ OCT parameter } \\
\hline pRNFL at baseline $(\mu \mathrm{m})^{c}$ & NA & $97.0(10.9)$ & NA & $97.2(8.9)$ \\
\hline pRNFL at last follow up $(\mu \mathrm{m})^{c}$ & NA & NA & $95.6(10.0)$ & $96.1(6.5)$ \\
\hline aLpRNFL $(\mu \mathrm{m} / \text { year })^{c}$ & NA & NA & NA & $-0.6(1.5)$ \\
\hline
\end{tabular}

aLPRNFL, annualized loss of peripapillary retinal nerve fiber layer; ARR, annualized relapse rate; DMT, disease modifying treatment; EDSS, Expanded Disability Status Scale; NA, not applicable; OCT, optical coherence tomography; PRNFL, peripapillary retinal nerve fiber layer.

${ }^{a}$ Number (percentage), ${ }^{b}$ Median and interquartile range, ${ }^{c}$ Mean and standard deviation.

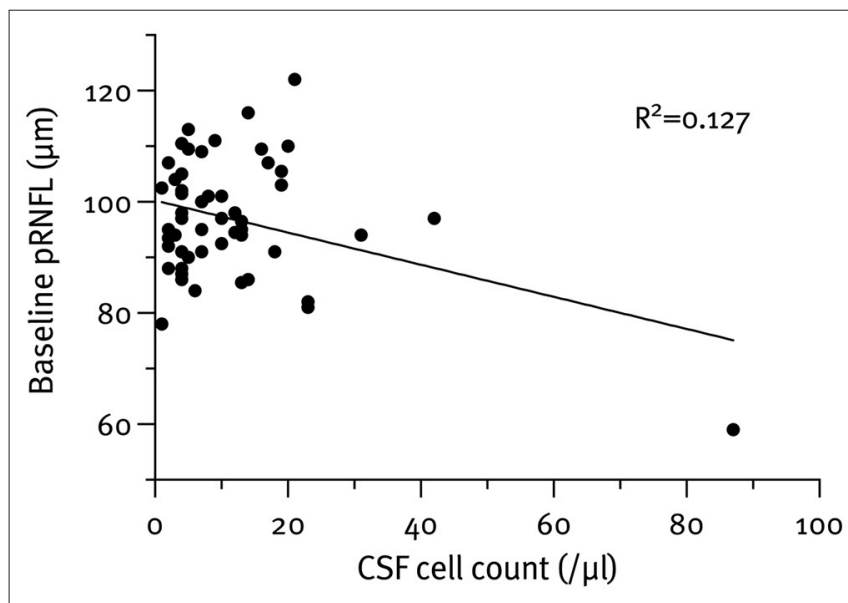

FIGURE 2 | Cerebrospinal fluid (CSF) cell count (WBC) correlated with the peripapillary retinal nerve fiber layer (pRNFL) thickness at baseline $(p=0.008)$.

Out of all classical CSF parameters investigated, WBC count emerged as the sole statistically significant predictor of $\mathrm{pRNFL}$ thickness at baseline. That being said, the effect size was moderate at best and only explained about $13 \%$ of the pRNFL thickness variation.

Importantly, the CSF cell count was not associated with pRNFL thickness at follow-up or thinning of pRNFL during follow-up. The observation that WBC count is associated with pRNFL thickness at the time of LP but not with further pRNFL thinning suggests that WBC count is reflective of short-term inflammatory activity rather than long-term disease progression, i.e., neuroaxonal damage. This is aligned with evidence indicating that increased WBC is associated with faster conversion from clinically isolated syndrome (CIS) to clinically definite MS but only very weakly with EDSS worsening $(29,30)$.

This was the first published study to investigate the associations between NfL in CSF and retinal layer thinning. We did not find any significant association, even though we observed a trend toward increased CSF NfL levels and both lower pRNFL thickness at baseline, as well as increased aLpRNFL. Previous studies have shown an association between NfL levels in serum and both pRNFL thickness and aLpRNFL (31). As serum and CSF NfL levels are tightly correlated, the lack of a significant association may be explained by various factors. Our 
TABLE 2 | Univariate correlation between cerebrospinal fluid (CSF) parameters and annualized loss of peripapillary retinal nerve fiber layer (aLpRNFL).

\begin{tabular}{|c|c|c|c|c|}
\hline & Study cohort $(n=61)$ & aLpRNFL $\geq 1.5 \mu \mathrm{m} / \mathrm{y}(n=6)$ & aLpRNFL $<1.5 \mu \mathrm{m} / \mathrm{y}(n=19)$ & Correlation with aLpRNFL \\
\hline WBC count $(/ \mu I)^{a}$ & $7(4-13.5)$ & $8(6.5-12.5)$ & $7(4-19)$ & $r_{s}=-0.025$ \\
\hline Total protein level $(\mathrm{mg} / \mathrm{dl})^{\mathrm{b}}$ & $35.6(12.4)$ & $39.9(11.1)$ & $35.0(17.3)$ & $r=-0.017$ \\
\hline Serum lgG level $(\mathrm{mg} / \mathrm{dll})^{\mathrm{b}}$ & 944.95 (180.42) & $855.33(223.40)$ & $955.42(162.73)$ & $r=-0.240$ \\
\hline CSF IgG level (mg/dl) ${ }^{a}$ & $3.33(2.19-4.52)$ & $3.19(2.13-4.16)$ & $3.53(1.56-6.39)$ & $r_{s}=-0.213$ \\
\hline IgG ratio $(/ 1,000)^{\mathrm{a}}$ & $3.57(2.65-4.92)$ & $3.46(3.16-4.10)$ & $3.57(1.78-6.46)$ & $r_{s}=-0.178$ \\
\hline $\operatorname{lgG}$ index ${ }^{a}$ & $0.70(0.61-1.12)$ & $0.64(0.55-0.94)$ & $0.76(0.66-1.23)$ & $r_{s}=-0.282$ \\
\hline Serum lgA level (mg/dl) ${ }^{a}$ & 153 (133-239.5) & 148.5 (106.3-379.3) & $204(115-271)$ & $r_{s}=-0.026$ \\
\hline CSF IgA level (mg/dl) ${ }^{a}$ & $0.48(0.27-0.62)$ & $0.25(0.14-0.90)$ & $0.46(0.12-0.98)$ & $r_{s}=-0.407$ \\
\hline IgA ratio $(/ 1,000)^{a}$ & $1.90(1.26-2.81)$ & $1.30(0.90-2.10)$ & $2.63(0.84-4.55)$ & $r_{s}=-0.629^{\star \star}$ \\
\hline $\lg A$ index ${ }^{a}$ & $0.32(0.24-0.44)$ & $0.23(0.22-0.30)$ & $0.30(0.26-0.70)$ & $r_{s}=-0.678^{\star \star *}$ \\
\hline Serum lgM level (mg/dl) ${ }^{a}$ & 105 (69.5-147) & $63.5(45.8-85.3)$ & $114(76-169)$ & $r_{s}=-0.176$ \\
\hline CSF IgM level (mg/dl) ${ }^{\mathrm{a}}$ & $0.06(0.03-0.08)$ & $0.05^{\star}$ & $0.03^{*}$ & $r_{s}=0.316$ \\
\hline IgM ratio $(/ 1,000)^{\mathrm{a}}$ & $0.44(0.27-0.87)$ & $0.54^{*}$ & $0.12^{\star}$ & $r_{s}=0.700$ \\
\hline IgM index ${ }^{a}$ & $0.08(0.06-0.18)$ & $0.12^{*}$ & $0.05^{\star}$ & $r_{s}=0.500$ \\
\hline Albumin ratio $(/ 1,000)^{a}$ & $4.85(3.12-6.18)$ & $5.14(3.66-7.58)$ & $3.26(2.65-5.69)$ & $r_{s}=0.102$ \\
\hline $\operatorname{csF} \mathrm{NfL}(\mathrm{pg} / \mathrm{ml})^{\mathrm{a}}$ & 798.8 (426.3-1,507.6) & $830.7(300.5-1,387.1)$ & $497.3(447.9-752.7)$ & $r_{s}=0.146$ \\
\hline sNfL $(p g / m l)^{a}$ & $10.6(7.1-20.2)$ & $6.9(4.8-45.8)$ & $8.0(4.3-12.6)$ & $r_{s}=0.159$ \\
\hline
\end{tabular}

aLpRNFL, annualized loss of peripapillary retinal nerve fiber layer; CSF, cerebrospinal fluid; NfL, neurofilament light chain; WBC, white blood cell.

${ }^{a}$ Median and interquartile range, ${ }^{b}$ Mean and standard deviation.

${ }^{\star}$ Sample size too small to calculate distributions, ${ }^{\star \star} p<0.05,{ }^{\star \star *} p<0.01$.

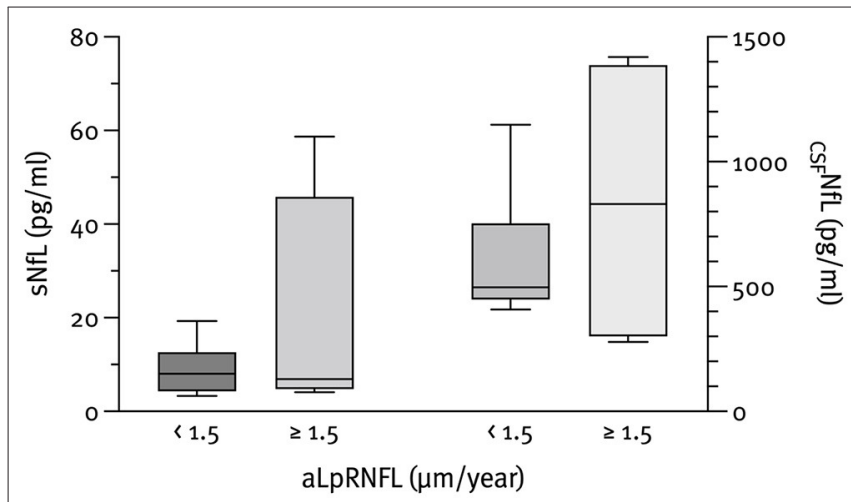

FIGURE 3 | Baseline CSF neurofilament light chain (CSFNfL) $(n=10)$ and serum neurofilament light chain ( $\mathrm{sNfL}$ ( $n=12)$ concentrations according to the rate of annualized loss of pRNFL (aLpRNFL). cohort consisted mostly of newly diagnosed patients, thus, the degree of neuroaxonal damage was inherently low, narrowing the potential margin of prediction. Also, the majority of patients $(29 / 38 ; 76.3 \%)$ were placed on DMT early on, which may negate the predictive capacity of NfL. Furthermore, it is important to bear in mind that NfL levels are somewhat volatile and one-time sampling combined with a low prognostic margin may lead to statistically unfavorable results (31).

Our results are limited with respect to the small sample size. Data on aLpRNFL was only available for 25 patients with only 6 of them showing signs of progression. We did not include GCIPL thickness which is reported to be even a more sensitive biomarker of disease progression in MS $(5,32,33)$. Also, paired serum and

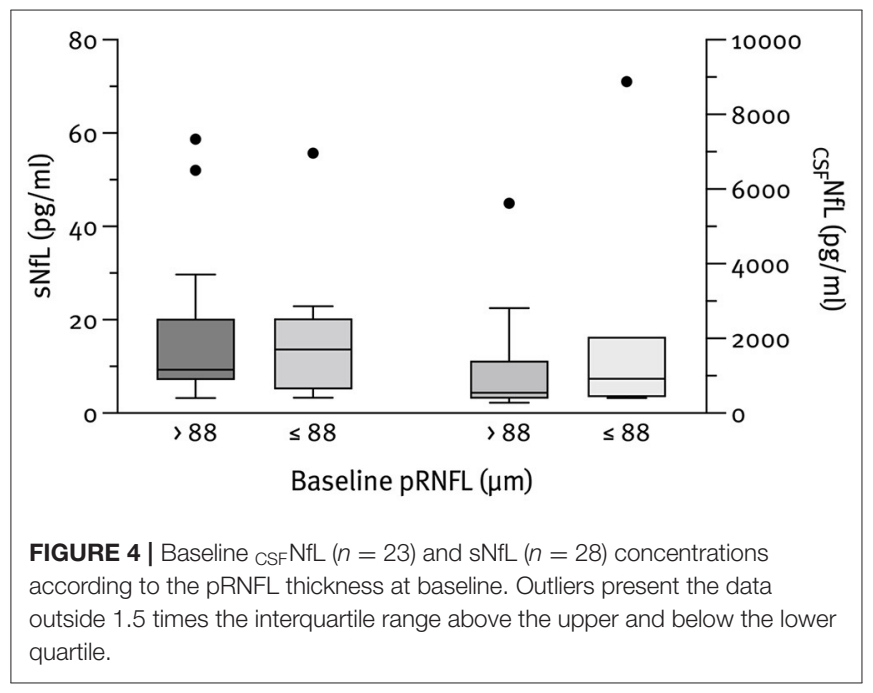

CSF samples for NfL analysis were not available for all subjects. We tried to overcome this predicament by performing sensitivity analyses for the missing NfL values and found no significant unfavorable effect. Besides, due to the lack of sufficient MRI data, the optic chiasm involvement was not excluded. However, the potential impact of optic chiasm involvement is probably low as this is-contrary to neuromyelitis optica spectrum disorderrare in MS.

In conclusion, we showed some evidence that from all CSF parameters obtained at diagnosis, the WBC count remained the only parameter to determine decreased retinal thickness. In our model, NfL concentrations in both serum and CSF 
upon diagnosis were not robust enough to predict subsequent retinal thinning.

\section{DATA AVAILABILITY STATEMENT}

The original contributions presented in the study are included in the article/supplementary material, further inquiries can be directed to the corresponding author/s.

\section{ETHICS STATEMENT}

The studies involving human participants were reviewed and approved by the Ethics Committee of the Medical University of Vienna, Austria (EK1446/2021). Written informed consent for participation was not required for this study in accordance with the national legislation and the institutional requirements.

\section{REFERENCES}

1. Compston A, Coles A. Multiple sclerosis. Lancet. (2002) 359:122131. doi: 10.1016/S0140-6736(02)08220-X

2. Hegen H, Bsteh G, Berger T. 'No evidence of disease activity' - is it an appropriate surrogate in multiple sclerosis? Eur J Neurol. (2018) 25:1107e101. doi: 10.1111/ene.13669

3. Britze J, Frederiksen JL. Optical coherence tomography in multiple sclerosis. Eye (Lond). (2018) 32:884-8. doi: 10.1038/s41433-017-0010-2

4. Saidha S, Sotirchos ES, Oh J, Syc SB, Seigo MA, Shiee N, et al. Relationships between retinal axonal and neuronal measures and global central nervous system pathology in multiple sclerosis. JAMA Neurol. (2013) 70:3443. doi: 10.1001/jamaneurol.2013.573

5. Bsteh G, Berek K, Hegen H, Altmann P, Wurth S, Auer M, et al. Macular ganglion cell-inner plexiform layer thinning as a biomarker of disability progression in relapsing multiple sclerosis. Mult Scler. (2021) 27:68494. doi: $10.1177 / 1352458520935724$

6. Bsteh G, Hegen H, Teuchner B, Berek K, Wurth S, Auer M, et al. Peripapillary retinal nerve fibre layer thinning rate as a biomarker discriminating stable and progressing relapsing-remitting multiple sclerosis. Eur J Neurol. (2019) 26:865-71. doi: 10.1111/ene.13897

7. Petzold A, Balcer LJ, Calabresi PA, Costello F, Frohman TC, Frohman EM, et al. Retinal layer segmentation in multiple sclerosis: a systematic review and meta-analysis. Lancet Neurol. (2017) 16:797-812. doi: 10.1016/S1474-4422(17)30278-8

8. Andersson M, Alvarez-Cermeno J, Bernardi G, Cogato I, Fredman P, Frederiksen J, et al. Cerebrospinal fluid in the diagnosis of multiple sclerosis: a consensus report. J Neurol Neurosurg Psychiatry. (1994) 57:897902. doi: 10.1136/jnnp.57.8.897

9. Link H. Immunoglobulin $\mathrm{G}$ and low molecular weight proteins in human cerebrospinal fluid. Chemical and immunological characterisation with special reference to multiple sclerosis. Acta Neurol Scand. (1967) 43:1-136.

10. McDonald WI, Compston A, Edan G, Goodkin D, Hartung HP, Lublin FD, et al. Recommended diagnostic criteria for multiple sclerosis: guidelines from the International Panel on the diagnosis of multiple sclerosis. Ann Neurol. (2001) 50:121-7. doi: 10.1002/ana.1032

11. Freedman MS, Thompson EJ, Deisenhammer F, Giovannoni G, Grimsley G, Keir G, et al. Recommended standard of cerebrospinal fluid analysis in the diagnosis of multiple sclerosis: a consensus statement. Arch Neurol. (2005) 62:865-70. doi: 10.1001/archneur.62.6.865

12. Schwid SR, Decker MD, Lopez-Bresnahan M, Rebif-Influenza Vaccine Study I. Immune response to influenza vaccine is maintained in patients with multiple sclerosis receiving interferon beta-1a. Neurology. (2005) 65:19646. doi: 10.1212/01.wnl.0000188901.12700.e0

\section{AUTHOR CONTRIBUTIONS}

NK: acquisition of data, data management, statistical analysis and interpretation of data, and drafting of the manuscript. PA: acquisition of data, data management, interpretation of data, and drafting of the manuscript. KR, CM, PR, FL, TB, and BP: acquisition of data and critical revision of the manuscript for intellectual content. GB: study concept and design, acquisition of data, interpretation of data, study supervision, and critical revision of the manuscript for intellectual content. All authors contributed to the article and approved the submitted version.

\section{FUNDING}

This research was funded by the Medical University of Vienna.

13. Khalil M, Teunissen CE, Otto M, Piehl F, Sormani MP, Gattringer T, et al. Neurofilaments as biomarkers in neurological disorders. Nat Rev Neurol. (2018) 14:577-89. doi: 10.1038/s41582-018-0058-Z

14. Barro C, Benkert P, Disanto G, Tsagkas C, Amann M, Naegelin Y, et al. Serum neurofilament as a predictor of disease worsening and brain and spinal cord atrophy in multiple sclerosis. Brain. (2018) 141:238291. doi: 10.1093/brain/awy154

15. Poser CM, Paty DW, Scheinberg L, McDonald WI, Davis FA, Ebers GC, et al. New diagnostic criteria for multiple sclerosis: guidelines for research protocols. Ann Neurol. (1983) 13:227-31. doi: 10.1002/ana.410130302

16. Kurtzke JF. Rating neurologic impairment in multiple sclerosis: an expanded disability status scale (EDSS). Neurology. (1983) 33:144452. doi: 10.1212/WNL.33.11.1444

17. Pemp B, Kardon RH, Kircher K, Pernicka E, Schmidt-Erfurth U, Reitner A. Effectiveness of averaging strategies to reduce variance in retinal nerve fibre layer thickness measurements using spectral-domain optical coherence tomography. Graefes Arch Clin Exp Ophthalmol. (2013) 251:18418. doi: 10.1007/s00417-013-2337-0

18. Tewarie P, Balk L, Costello F, Green A, Martin R, Schippling S, et al. The OSCAR-IB consensus criteria for retinal OCT quality assessment. PLoS ONE. (2012) 7:e34823. doi: 10.1371/journal.pone.0034823

19. Nolan-Kenney RC, Liu M, Akhand O, Calabresi PA, Paul F, Petzold A, et al. Optimal intereye difference thresholds by optical coherence tomography in multiple sclerosis: An international study. Ann Neurol. (2019) 85:61829. doi: 10.1002/ana.25462

20. Bsteh G, Hegen H, Altmann P, Auer M, Berek K, Zinganell A, et al. Validation of inter-eye difference thresholds in optical coherence tomography for identification of optic neuritis in multiple sclerosis. Mult Scler Relat Disord. (2020) 45:102403. doi: 10.1016/j.msard.2020.102403

21. Bsteh G, Hegen H, Teuchner B, Amprosi M, Berek K, Ladstatter F, et al. Peripapillary retinal nerve fibre layer as measured by optical coherence tomography is a prognostic biomarker not only for physical but also for cognitive disability progression in multiple sclerosis. Mult Scler. (2019) 25:196-203. doi: 10.1177/1352458517740216

22. Saidha S, Al-Louzi O, Ratchford JN, Bhargava P, Oh J, Newsome SD, et al. Optical coherence tomography reflects brain atrophy in multiple sclerosis: A four-year study. Ann Neurol. (2015) 78:801-13. doi: 10.1002/ana.24487

23. Aytulun A, Cruz-Herranz A, Aktas O, Balcer LJ, Balk L, Barboni P. et al. APOSTEL 20 recommendations for reporting quantitative optical coherence tomography studies. Neurology. (2021) 97:68-79. doi: 10.1212/WNL.0000000000012125

24. Altmann P, Ponleitner M, Rommer PS, Haslacher H, Mucher P, Leutmezer F, et al. Seven day pre-analytical stability of serum and plasma neurofilament light chain. Sci Rep. (2021) 11:11034. doi: 10.1038/s41598-021-90639-z 
25. Haslacher H, Gerner M, Hofer P, Jurkowitsch A, Hainfellner J, Kain R, et al. Usage Data and Scientific Impact of the Prospectively Established Fluid Bioresources at the Hospital-Based MedUni Wien Biobank. Biopreserv Biobank. (2018) 16:477-82. doi: 10.1089/bio.2018.0032

26. Abalo-Lojo JM, Limeres CC, Gomez MA, Baleato-Gonzalez S, Cadarso-Suarez C, Capeans-Tome C, et al. Retinal nerve fiber layer thickness, brain atrophy, and disability in multiple sclerosis patients. J Neuroophthalmol. (2014) 34:238. doi: 10.1097/WNO.0000000000000057

27. Ratchford JN, Saidha S, Sotirchos ES, Oh JA, Seigo MA, Eckstein C, et al. Active MS is associated with accelerated retinal ganglion cell/inner plexiform layer thinning. Neurology. (2013) 80:47-54. doi: 10.1212/WNL.0b013e31827b1a1c

28. Coric D, Balk LJ, Verrijp M, Eijlers A, Schoonheim MM, Killestein J, et al. Cognitive impairment in patients with multiple sclerosis is associated with atrophy of the inner retinal layers. Mult Scler. (2018) 24:15866. doi: 10.1177/1352458517694090

29. Berek K, Bsteh G, Auer M, Di Pauli F, Zinganell A, Berger T, et al. Cerebrospinal Fluid Findings in 541 Patients With Clinically Isolated Syndrome and Multiple Sclerosis: A Monocentric Study. Front Immunol. (2021) 12:675307. doi: 10.3389/fimmu.2021.675307

30. Lotan I, Benninger F, Mendel R, Hellmann MA, Steiner I. Does CSF pleocytosis have a predictive value for disease course in MS? Neurol Neuroimmunol Neuroinflamm. (2019) 6:e584. doi: 10.1212/NXI.0000000000000584

31. Bsteh G, Berek K, Hegen H, Teuchner B, Buchmann A, Voortman $\mathrm{MM}$, et al. Serum neurofilament levels correlate with retinal nerve fiber layer thinning in multiple sclerosis. Mult Scler. (2020) 26:168290. doi: $10.1177 / 1352458519882279$

32. Schurz N, Sariaslani L, Altmann P, Leutmezer F, Mitsch C, Pemp B, et al. Evaluation of Retinal Layer Thickness Parameters as Biomarkers in a Real-World Multiple Sclerosis Cohort. Eye Brain. (2021) 13:5969. doi: 10.2147/EB.S295610

33. Bsteh G, Hegen H, Altmann P, Auer M, Berek K, Pauli FD, et al. Retinal layer thinning is reflecting disability progression independent of relapse activity in multiple sclerosis. Mult Scler J Exp Transl Clin. (2020) 6:2055217320966344. doi: 10.1177/2055217320966344

Conflict of Interest: NK has participated in meetings sponsored by, received speaker honoraria or travel funding from Roche, Novartis, and Merck, and holds a grant for a Multiple Sclerosis Clinical Training Fellowship Programme from the European Committee for Treatment and Research in Multiple Sclerosis (ECTRIMS). PA has participated in meetings sponsored by, received speaker honoraria or travel funding from Biogen, Merck, Roche, Sanofi-Genzyme and Teva, and received honoraria for consulting from Biogen. He received a research grant from Quanterix International and was awarded a combined sponsorship from Biogen, Merck, Sanofi-Genzyme, Roche, and Teva for a clinical study. CM has received honoraria for consultancy/speaking (incl. funds for e-learning modules) from Bayer, Novartis, and Takeda. PR has received honoraria for consultancy/speaking from AbbVie, Allmiral, Alexion, Biogen, Merck, Novartis, Roche, Sandoz, Sanofi Genzyme, has received research grants from Amicus, Biogen, Merck, Roche. FL received honoraria (lectures, advisory boards, consultations) from pharmaceutical companies marketing treatments for MS: Alexion, Almirall, Bayer, Biogen, Celgene, MedDay, Merck, Novartis, Octapharma, Roche, Sanofi-Genzyme, Teva. TB has participated in meetings sponsored by and received honoraria (lectures, advisory boards, consultations) from pharmaceutical companies marketing treatments for MS: Almirall, Biogen, Bionorica, Celgene/BMS, GSK, Janssen-Cilag, MedDay, Merck, Novartis, Roche, Sandoz, Sanofi-Genzyme, Teva. His institution has received financial support in the past 12 months by unrestricted research grants Biogen, Celgene/BMS, Merck, Novartis, Sanofi Aventis, Teva and for participation in clinical trials in multiple sclerosis sponsored by Alexion, Biogen, Merck, Novartis, Octapharma, Roche, Sanofi-Genzyme, Teva. BP has received honoraria for consulting from Novartis. GB has participated in meetings sponsored by, received speaker honoraria or travel funding from Biogen, Celgene, Lilly, Merck, Novartis, Sanofi-Genzyme, and Teva, and received honoraria for consulting Biogen, Celgene, Novartis, Sanofi-Genzyme, Roche, and Teva.

The remaining author declares that the research was conducted in the absence of any commercial or financial relationships that could be construed as a potential conflict of interest.

Publisher's Note: All claims expressed in this article are solely those of the authors and do not necessarily represent those of their affiliated organizations, or those of the publisher, the editors and the reviewers. Any product that may be evaluated in this article, or claim that may be made by its manufacturer, is not guaranteed or endorsed by the publisher.

Copyright (c) 2022 Krajnc, Altmann, Riedl, Mitsch, Berger, Leutmezer, Rommer, Pemp and Bsteh. This is an open-access article distributed under the terms of the Creative Commons Attribution License (CC BY). The use, distribution or reproduction in other forums is permitted, provided the original author(s) and the copyright owner(s) are credited and that the original publication in this journal is cited, in accordance with accepted academic practice. No use, distribution or reproduction is permitted which does not comply with these terms. 